\title{
Desenvolvimento e avaliação físico-química, sensorial e da estabilidade de ácido ascórbico de doce de leite com pimenta
}

\author{
Development of red pepper-containing dulce de leche and evaluation \\ of its physico-chemical and sensorial characteristics, and ascorbic acid \\ stability
}

RIALA6/1555

Camila Pegorer MAZINI ${ }^{1}$, Gabriella Giani PIERETTI ${ }^{1}$, Ivanise Guilherme BRANCO², Monica Regina

da Silva SCAPIM ${ }^{1}$, Grasiele Scaramal MADRONA ${ }^{1^{*}}$

*Endereço para correspondência: ${ }^{1}$ Laboratório de Tecnologia de Leites, Departamento de Engenharia de Alimentos, Universidade Estadual de Maringá, Campus Maringá, CEP: 87020-900, Maringá, PR, Brasil, E-mail: gsmadrona@uem.br ${ }^{2}$ Faculdade de Ciências e Letras de Assis, Universidade Estadual Paulista Júlio de Mesquita Filho, Assis, SP, Brasil

Recebido: 22.01.2013 - Aceito para publicação: 30.06.2013

\section{RESUMO}

O doce de leite é um alimento muito consumido em todo o Brasil, com sabor, cor, aroma e texturas características. A pimenta dedo-de-moça também está presente na mesa dos consumidores e é rica em ácido ascórbico (vitamina C), que é excelente antioxidante. No presente trabalho, foi elaborado o doce de leite adicionado de pimenta dedo-de-moça. No produto desenvolvido, foi determinada a composição centesimal, e foi avaliada a aceitação do doce quanto aos atributos sensoriais de cor, aroma, sabor, textura e aceitação global. O teor de ácido ascórbico foi quantificado em diferentes períodos de armazenamento (0, 30 e 60 dias). O doce de leite com pimenta preparado apresentou composição centesimal de acordo com a legislação vigente. $\mathrm{O}$ teor de ácido ascórbico mostrou-se praticamente estável durante o período de armazenamento, e o produto demonstrou boa aceitação na análise sensorial.

Palavras-chave. doce de leite, pimenta dedo-de-moça, ácido ascórbico.

\begin{abstract}
The dulce de leche or milk candy is a food widely consumed in Brazil, which shows typical characteristics of taste, color, flavor and texture. The lady-finger red pepper is regularly present on the Brazilian people table as this product contains a pungent flavor favoring various spices; and it is also rich in ascorbic acid (vitamin C) which is an excellent antioxidant. This study aimed at developing a dulce de leche containing lady-finger red pepper, and to determine the composition of the product. Also, the ascorbic acid contents were quantified in products stored for different periods (0,30 and 60 days). The product acceptance by consumers on the sensory characteristics was evaluated by means of hedonic scale for color, aroma, flavor and texture attributes. The composition of the product was in accordance with the legislation in force, and the ascorbic acid contents were practically stable during the storage, and the sensory analyses indicated that the red pepper-containing dulce de leche was well accepted.
\end{abstract}

Keywords. dulce de leche, lady-finger red pepper, ascorbic acid. 


\section{INTRODUÇÃO}

O doce de leite é um alimento muito consumido na América Latina, em especial no Brasil e na Argentina ${ }^{1}$. Segundo Della Lucia et $\mathrm{al}^{2}$, é muito consumido como ingrediente para confeitos, bolos, sorvetes e também como sobremesa ou no acompanhamento de queijo, pão ou biscoito.

$\mathrm{Na}$ fabricação do doce, deve-se fazer uso de leite de qualidade, para proporcionar um doce de boa qualidade. Um dos problemas que devem ser controlados é a estabilidade microbiológica, tecnológica e física ${ }^{3}$.

O desenvolvimento de novos produtos pode manter pequenas indústrias em um mercado cada vez mais competitivo. Sendo assim, a indústria de laticínios tem buscado alternativas como novas matérias-primas na elaboração de seus produtos. Nesse contexto, mostra-se interessante o desenvolvimento de um produto diferenciado, por exemplo, um doce de leite com pimenta.

Pimentas vermelhas, como a pimenta dedode-moça do gênero Capcicum, são muito utilizadas como temperos para diversos pratos, principalmente pelo sabor pungente ("picante") que apresentam. As características específicas dessas pimentas se devem às substâncias, como a capsaicina, os carotenoides e o ácido ascórbico. A presença do ácido ascórbico, confere às pimentas poder antioxidante considerável ${ }^{4}$, que pode prevenir ou reduzir a oxidação de lipídios, proteínas e ácidos nucleicos causados por espécies de oxigênio reativo, que incluem os radicais livres que ao reagir com estes, impede os efeitos ruins ao organismo.

O tempo de armazenamento do doce de leite é relativamente longo devido ao açúcar, que além de atuar no sabor, corpo e cor do doce, é um conservante. O maior problema que o doce apresenta ao longo do tempo de armazenamento é a cristalização, que decorre da formação de grandes cristais de lactose ${ }^{3}$.

Por apresentar efeito antioxidante, o ácido ascórbico pode ser utilizado como conservante em alimentos, tendo ainda a vantagem de ser natural ${ }^{6}$. Ao adicionar a pimenta dedo-de-moça ao doce de leite, atribui-se o conservante natural e ainda aproveita-se o sabor pungente e contraditório ao doce, tornando-o, assim, um produto novo e com sabor diferenciado.

Nesse contexto, o presente trabalho tem como objetivo desenvolver e analisar sensorial e físico-quimicamente o doce de leite com pimenta dedo-de-moça, além de quantificar a disponibilidade do ácido ascórbico presente no produto durante o armazenamento.

\section{MATERIAL E MÉTODOS}

\section{Processamento e formulação do doce de leite com pimenta}

A metodologia empregada na produção do doce de leite com pimenta é a mesma descrita por Hosken $^{7}$, sendo modificada apenas na última etapa, na qual é acrescentada a pimenta dedo-de-moça, que foi previamente higienizada em solução de 100 ppm de cloro por 20 minutos, enxaguada, as sementes retiradas e, em seguida, trituradas.

Após adição dos ingredientes, iniciou-se o processo de concentração do doce de leite em tacho aberto, com agitação até $65^{\circ}$ Brix, que foi medido em triplicatas por um refratômetro de mesa com leitura refratométrica a $20{ }^{\circ} \mathrm{C}$. Após atingir a concentração desejada, foi realizado o resfriamento à $70{ }^{\circ} \mathrm{C}$, depois realizou-se o envase em potes de vidro e, por último, um resfriamento até temperatura ambiente.

Foram testadas, previamente, diferentes concentrações de pimenta e, ao final, foram selecionadas três formulações, cujas porcentagens dos ingredientes estão apresentadas na Tabela 1.

Tabela 1. Ingredientes das formulações de doce de leite com pimenta

\begin{tabular}{cccc}
\hline Ingredientes & $\mathbf{1 \%}$ & $\mathbf{2} \%$ & $\mathbf{3} \%$ \\
\hline Leite (\%) & 79,75 & 79,75 & 79,75 \\
Açúcar (\%) & 19,94 & 19,94 & 19,94 \\
Amido (\%) & 0,25 & 0,25 & 0,25 \\
Bicarbonato de sódio (\%) & 0,06 & 0,06 & 0,06 \\
Pimenta dedo-de-moça (\%) & 1 & 2 & 3 \\
\hline Total (\%) & 100 & 100 & 100 \\
\hline
\end{tabular}

\section{Análises físico-químicas}

Os teores de umidade, gorduras totais e cinzas foram determinados por metodologia descrita pelo Instituto Adolfo Lutz ${ }^{8}$. Na determinação do teor de proteínas, foi utilizado o método segundo o manual AOAC 9 .

A quantificação do ácido ascórbico foi feita pelo método descrito pelo Instituto Adolfo Lutz ${ }^{10}$. Análises de ácido ascórbico no produto foram realizadas em diferentes períodos ( 0,30 e 60 dias), para verificar sua estabilidade durante $\mathrm{o}$ armazenamento. 


\section{Análise de cor e textura instrumental}

A cor do doce de leite com pimenta foi avaliada por meio de um colorímetro portátil Minolta ${ }^{\oplus}$ CR10, com esfera de integração e ângulo de visão de $3^{\circ}$, ou seja, iluminação d/3 e iluminante D65. O sistema utilizado foi o CIEL ${ }^{*} a^{*} b^{*}$, com o qual foram medidas as coordenadas: $L^{*}$, representando a luminosidade em uma escala de 0 (preto) a 100 (branco); $\mathrm{a}^{*}$ que representa uma escala de tonalidade variando de vermelho $(0+a)$ a verde $(0-a)$ e $b^{\star}$ que representa uma escala de amarelo $(0+b)$ a azul $(0$ - b). Todas as determinações foram feitas em triplicata.

A análise de textura do produto foi realizada em Texturômetro modeloStable MicroSystems Texture Analyser TAXT Plus (Texture Technologies Corp, Inglaterra). As características do ensaio foram: Acessório: Probe $36 \mathrm{~mm}$; Modo: força medida em compressão; Opção: retornar ao início; Velocidade de pré-teste: $2,0 \mathrm{~mm} / \mathrm{s}$; Velocidade de teste: $3,0 \mathrm{~mm} / \mathrm{s}$; Velocidade de pós-teste: 7,0 mm/s; Distância: 10 $\mathrm{mm}$. Essa análise foi realizada em duplicata.

\section{Análise sensorial}

$\mathrm{Na}$ avaliação da aceitação do doce de leite com pimenta foi utilizada escala hedônica de 1 (desgostei muitíssimo) a 9 pontos (gostei muitíssimo). A análise foi realizada no laboratório de Análise Sensorial do Departamento de Engenharia de Alimentos da Universidade Estadual de Maringá, com 101 provadores não treinados de ambos os sexos, com o parecer aprovado pelo comitê de ética da UEM com número CAAE: 02643312.4.0000.0104.

Foram avaliados os atributos cor, aroma, sabor, textura e aparência global. As amostras foram servidas puras em copos descartáveis codificados com números de três dígitos, acompanhadas de água para ser intercalada entre as amostras.

A intenção de compra foi avaliada em escala de 1 (certamente não compraria) a 3 (certamente compraria). Foi calculado o índice de aceitação do doce de leite com pimenta através da fórmula:

$$
I A=\frac{X * 100}{N}
$$

Onde:

$\mathrm{X}=$ média da amostra;

$\mathrm{N}=$ nota máxima da amostra dada pelos provadores

\section{Análise dos dados}

A análise estatística dos resultados foi realizada utilizando-se análise de variância (ANOVA) e cálculo de médias por Tukey a $5 \%$ de significância, teste que deve ser aplicado toda vez que se pretende comparar as médias dos tratamentos.

\section{RESULTADOS}

\section{Composição Centesimal}

$\mathrm{Na}$ Tabela 2 são apresentados os resultados das análises de umidade, cinzas, proteínas, gorduras totais, ácido ascórbico e ainda os valores de referência da legislação vigente no tempo zero.

Tabela 2. Tabela de composição centesimal em comparação à legislação vigente no tempo zero

\begin{tabular}{ccccc}
\hline Análises & Legislação $^{* *}$ & $\mathbf{1 \%}$ & $\mathbf{2} \%$ & $\mathbf{3 \%}$ \\
\hline \multirow{2}{*}{ Umidade (\%) } & máx. 30,0\% & $28,12 \pm$ & $29,60 \pm$ & $30,06 \pm$ \\
& & $0,0072^{\mathrm{a}}$ & $0,0648^{\mathrm{a}}$ & $0,0840^{\mathrm{a}}$ \\
Cinzas (\%) & máx. 2,0 \% & $1,58 \pm$ & $1,47 \pm$ & $1,36 \pm$ \\
& & $0,00005^{\mathrm{a}}$ & $0,00005^{\mathrm{a}}$ & $0,00245^{\mathrm{a}}$ \\
Proteínas (\%) & mín. 6,0\% & $6,40 \pm$ & $6,16 \pm$ & $6,16 \pm$ \\
Gorduras & \multirow{2}{*}{ mín. 2,0\% } & $5,00063^{\mathrm{a}}$ & $0,00000^{\mathrm{a}}$ & $0,00000^{\mathrm{a}}$ \\
Totais (\%) & & $0,0002^{\mathrm{a}}$ & $0,0049^{\mathrm{a}}$ & $0,00003^{\mathrm{a}}$ \\
Carboidratos & & 51,95 & 50,05 & 48,51 \\
$(\%)$ & - & $5,61 \pm$ & \\
\hline
\end{tabular}

${ }^{*}$ Letras iguais na mesma linha não diferem entre si ao nível de $5 \%$ de significância

** BRASIL. Ministério da Agricultura e do Abastecimento, Portaria No 354 , de 4 de setembro de $1997^{11}$

Pode-se verificar que não houve diferença significativa, ao nível de $5 \%$ de significância, entre as diferentes formulações para todas as análises, mostrando que a adição de até $3 \%$ de pimenta dedo-demoça não acarreta alterações na composição centesimal do doce de leite.

Em comparação a legislação vigente, ou seja, Portaria $n^{\circ} 354 / 97^{11}$, nota-se que o novo produto, para as 3 formulações testadas, está dentro dos padrões exigidos.

Segundo Feihrmann et $\mathrm{al}^{12}$, que também determinaram a composição centesimal de doce de leite pastoso com leite semidesnatado elaborado em um evaporador, obtiveram umidade de 33,4 \%, lipídeos de $4,75 \%$, proteínas de $10,2 \%$ e cinzas de 2,41\%. Comparando com os autores, o doce de leite em estudo apresenta poucas diferenças na composição centesimal. É importante ressaltar que o leite utilizado pelos autores foi semidesnatado e, para elaboração do doce de leite com pimenta, foi utilizado o leite integral, o que explica a variação de gorduras/lipídeos e proteínas apresentada. 
A composição química de vários doces de leite adquiridos no comércio de São Paulo e Paraná foi avaliada por Demiate et $\mathrm{al}^{1}$. Os autores observaram teor médio de $26,80 \%$ de umidade, $1,41 \%$ de cinzas, 3,90 \% de lipídeos e $7,07 \%$ de proteínas que, comparados ao trabalho proposto, apresentaram poucas variações. Essas pequenas variações podem ser explicadas pela tecnologia utilizada na fabricação dos doces de leite comerciais.

\section{Estabilidade do ácido ascórbico}

O ácido ascórbico foi quantificado nas amostras de doce de leite no período de 2 meses, sendo realizadas análises no tempo 0, 30 e 60 dias. A Tabela 3 apresenta os resultados dessas análises nos diferentes tempos.

Não houve diferença significativa entre o teor de ácido ascórbico presente nas amostras, ao nível de $5 \%$ de significância, ao longo do período de armazenamento (Tabela 3).

Esses resultados indicam que se o doce de leite for armazenado de maneira correta, o teor de ácido ascórbico presente na pimenta continuará estável, proporcionando um doce de leite com pimenta de valor considerável de vitamina $\mathrm{C}$, no período de 60 dias, conforme analisado.

Tabela 3. Concentração (mg/100g de produto) de ácido ascórbico durante o tempo de armazenamento

\begin{tabular}{cccc}
$\begin{array}{c}\text { Tempo } \\
(\text { dias })\end{array}$ & $1 \%$ & $2 \%$ & $\mathbf{3} \%$ \\
\hline 0 & $6,79 \pm 0,05^{\mathrm{aA}}$ & $8,11 \pm 0,23^{\mathrm{aA}}$ & $8,50 \pm 0,06^{\mathrm{aA}}$ \\
30 & $6,45 \pm 0,22^{\mathrm{aA}}$ & $6,21 \pm 0,00^{\mathrm{aA}}$ & $8,12 \pm 0,23^{\mathrm{aA}}$ \\
60 & $6,83 \pm 0,02^{\mathrm{aA}}$ & $5,99 \pm 0,05^{\mathrm{aA}}$ & $8,55 \pm 0,23^{\mathrm{aA}}$ \\
\hline
\end{tabular}

${ }^{\star}$ Letras iguais na mesma linha não diferem entre si ao nível de $5 \%$ de significância

${ }^{*}$ Letras iguais maiúsculas na mesma coluna não diferem entre si ao nível de $5 \%$ de significância.

\section{Cor e textura}

$\mathrm{Na}$ Tabela 4, são apresentados os resultados da análise de cor para as três formulações.

Pode-se observar que os resultados da análise de cor $\left(L, a^{\star}\right.$ e $\left.b^{\star}\right)$ não diferem entre si, ao nível de $5 \%$ de significância para as diferentes concentrações de pimenta. Isso demonstra que a adição de até $3 \%$ de pimenta dedo-de-moça não interfere na cor do doce de leite nas concentrações avaliadas.

Bellarde $\mathrm{e}^{13}$ analisou instrumentalmente a cor de doce de leite (com mesmo teor em ${ }^{\circ}$ Brix), obtendo para "L" o valor de 50,77 , para "a*" 7,45 , e para " $\mathrm{b}^{\star}$ " 24,21. Comparando esses resultados com o doce de leite com pimenta, nota-se poucas variações desses parâmetros.
Observa-se na Tabela 4 que não houve diferença significativa entre os resultados de textura. Isso se deve ao fato de que a formulação do doce de leite era a mesma para todas as concentrações, já que a variação de pimenta adicionada depois do doce pronto foi pequena.

Tabela 4. Tabela de resultados da análise de cor de amostras de doce de leite acrescido de diferentes teores de pimenta dedo-de-moça

\begin{tabular}{cccr}
\hline \multirow{2}{*}{ Parâmetros } & \multicolumn{3}{c}{ Concentração de pimenta dedo-de-moça } \\
\cline { 2 - 4 } & $\mathbf{1 \%}$ & $\mathbf{2} \%$ & $\mathbf{3} \%$ \\
\hline L & $57,51 \pm 6,71^{\mathrm{a}}$ & $53,69 \pm 5,24^{\mathrm{a}}$ & $53,56 \pm 3,87^{\mathrm{a}}$ \\
$\mathrm{A}$ & $5,13 \pm 0,56^{\mathrm{a}}$ & $5,34 \pm 0,14^{\mathrm{a}}$ & $6,61 \pm 0,58^{\mathrm{a}}$ \\
B & $23,71 \pm 9,86^{\mathrm{a}}$ & $20,32 \pm 2,64^{\mathrm{a}}$ & $21,15 \pm 0,64^{\mathrm{a}}$ \\
Textura & $952,45 \pm$ & $1339,05 \pm$ & \\
(Kgf) & $220,005^{\mathrm{a}}$ & $125,044^{\mathrm{a}}$ & $1005,9 \pm 56,179^{\mathrm{a}}$ \\
\hline
\end{tabular}

${ }^{*}$ Letras iguais na mesma linha não diferem entre si ao nível de 5 \% de significância

\section{Análise Sensorial}

Os resultados da análise sensorial das diferentes formulações de doce de leite com pimenta estão na Tabela 5.

Observa-se na Tabela 5 que não houve diferença significativa entre as amostras, ao nível de $5 \%$ de significância, para todos os atributos avaliados.

É importante ressaltar que a análise sensorial foi realizada com provadores não treinados, que gostavam ou não de pimenta. $\mathrm{O}$ sabor pungente da pimenta não agrada a todos, ainda mais adicionado a um doce, contrapondo o característico sabor de doce de leite.

Para um produto ser bem aceito, o índice de aceitação deve ser superior a $70 \%$, assim, todas as formulações foram bem aceitas, apresentando índices que variam de $76,89 \%$ (para formulação com $3 \%$ ) e $84,04 \%$ (para formulação com $1 \%$ ).

Madrona et $\mathrm{al}^{14}$ realizaram análise sensorial de doces de leite elaborados com diferentes concentrações de soro de leite em substituição ao leite, comparando com o doce de leite tradicional (sem adição de soro), e obtiveram

Tabela 5. Resultados da análise sensorial do doce de leite com pimenta dedo-de-moça

\begin{tabular}{cccc}
\hline Atributos & $\mathbf{1} \%$ & $\mathbf{2} \%$ & $\mathbf{3 \%}$ \\
\hline Cor & $7,44 \pm 1,89^{\mathrm{a}}$ & $7,31 \pm 1,96^{\mathrm{a}}$ & $7,29 \pm 2,33^{\mathrm{a}}$ \\
Aroma & $7,41 \pm 1,76^{\mathrm{a}}$ & $7,02 \pm 2,40^{\mathrm{a}}$ & $7,05 \pm 2,21^{\mathrm{a}}$ \\
Sabor & $6,93 \pm 3,26^{\mathrm{a}}$ & $6,15 \pm 4,60^{\mathrm{a}}$ & $5,57 \pm 5,95^{\mathrm{a}}$ \\
Textura & $7,32 \pm 2,54^{\mathrm{a}}$ & $7,36 \pm 2,15^{\mathrm{a}}$ & $7,15 \pm 2,61^{\mathrm{a}}$ \\
Aparência Global & $7,56 \pm 1,65^{\mathrm{a}}$ & $7,22 \pm 2,02^{\mathrm{a}}$ & $6,92 \pm 3,23^{\mathrm{a}}$ \\
Intenção de Compra & $2,22 \pm 0,45^{\mathrm{a}}$ & $1,86 \pm 0,51^{\mathrm{a}}$ & $1,72 \pm 0,56^{\mathrm{a}}$ \\
Índice de aceitação & $84,04 \%$ & $80,30 \%$ & $76,89 \%$ \\
\hline
\end{tabular}

${ }^{*}$ Letras iguais na mesma linha não diferem entre si ao nível de $5 \%$ de significância 
média 6,6 no aspecto geral para o doce de leite sem adição de soro. O doce de leite com pimenta obteve as seguintes notas na avaliação global: 7,56 para concentração de $1 \%$ de pimenta, 7,22 para $2 \%$ de pimenta e 6,92 para $3 \%$ de pimenta. Comparando os resultados obtidos pelos autores com presente trabalho, pode-se dizer que o doce de leite com pimenta apresentou aparência global similar ao doce de leite tradicional, sendo favorável para comercialização.

Em relação à intenção de compra, 34,95 \% dos provadores certamente comprariam o doce de leite com $1 \%$ de pimenta dedo-de-moça, $51,45 \%$ talvez comprariam e $13,59 \%$ certamente não comprariam. Para o doce com $2 \%$ de pimenta, observamos que $20,38 \%$ dos provadores certamente comprariam, 46,60 \% talvez comprariam, e $33,01 \%$ certamente não comprariam. Para a formulação com $3 \%$ de pimenta, $17,47 \%$ dos provadores certamente comprariam, 36,89 \% talvez comprariam, e 45,63\% certamente não comprariam. Esses resultados indicam que todas as formulações são estatisticamente iguais e, ainda, que todas foram bem aceitas, apresentando índices de aceitação superiores a 76,89 \%.

Em geral, pode-se observar que os doces avaliados apresentaram alto índice de aceitação, assim como já observado na literatura. Feihrmann et $\mathrm{l}^{12}$, que produziram um doce de leite com leite semidesnatado concentrado e realizaram um teste de preferência, observaram que o produto foi aceito por $93 \%$ dos provadores, indicando que houve um bom índice de aceitação do produto.

\section{CONCLUSÃO}

O doce de leite com pimenta dedo-de-moça foi bem aceito na análise sensorial, sendo que os provadores não notaram diferença entre as diferentes concentrações de pimenta para os atributos avaliados. Na intenção de compra, as formulações apresentaram-se estatisticamente iguais. O ácido ascórbico presente no doce de leite manteve-se estável durante o período armazenamento.

\section{REFERÊNCIAS}

1. Demiate IM, Konkel FE, Pedroso RA. Avaliação da qualidade de amostras comerciais de doce de leite pastoso - composição química. Ciênc Tecnol Aliment. 2001;21(1):108-14.

2. Della Lucia AASM, Gomes ED, Nachtigall AM, Cavalcante JFM, Minim VPR. Perfil sensorial de doce de leite pastoso. Rev Inst Latic Cândido Tostes. 2003;334:45-50.

3. Coelho EBB, Coelho DT, Pinheiro AJR, Chaves JBP, Pereira AS. Utilização da beta-d-galactosidade no controle da cristalização do doce de leite. Rev Inst Latic Cândido Tostes. 1982;37:7-9.

4. Kappel VD. Avaliação das propriedades antioxidantes e antimicrobianas de extratos de Capsicum baccatum L. var. pendulum [dissertação de mestrado]. Porto Alegre (RS): Instituto de Ciências Básicas da Saúde, Universidade Federal do Rio Grande do Sul (UFRGS); 2007.

5. Couto MAL, Brazaca SGC. Quantificação de vitamina C e capacidade antioxidante de variedades cítricas. Ciênc Tecnol Aliment. 2010;30(1):15-9.

6. Dutra FLA, Branco IG, Madrona GS, Haminuk CI. Avaliação sensorial e influência do tratamento térmico no teor de ácido ascórbico de sorvete de pimenta. Rev Bras Tecnol Agroind. 2010;4(2):243-51.

7. Hosken FS. Doce de leite - Durabilidade e cristalização. Rev Inst Latic Cândido Tostes. 1969;24(147):10-7.

8. Instituto Adolfo Lutz. Normas Analíticas do Instituto Adolfo Lutz. Métodos químicos e físicos para a análise de alimentos, $4^{\mathrm{a}}$ Ed. São Paulo: IMESP, 2008.

9. Association of Official Analytical Chemists. Official Methods of Analysis, 16th ed., Washington, 1999.

10. Instituto Adolfo Lutz. Normas Analíticas do Instituto Adolfo Lutz. V. 1: Métodos químicos e físicos para a análise de alimentos, 3a Ed. São Paulo: IMESP, 1985.

11. Brasil. Ministério da Agricultura e do Abastecimento. Regulamento técnico para fixação de identidade e qualidade de doce de leite. Portaria no 354, de 04/09/97. Diário Oficial [da] União, Brasília, DF, 08 set. 1997. Seção I, p. 19685.

12. Feirmann AC, Cichoski AJ, Jacques AR. Doce de leite elaborado em evaporador, com leite semidesnatado concentrado. Hig Alim. 2006;20(141):29-32.

13. Bellarde FB. Elaboração de doce de leite pastoso com substituição parcial de sólidos do leite por concentrado proteico do soro. Rev Uniar. 2006;17(18):249-55.

14. Madrona GS, Zotarelli MF, Bergamasco R, Branco IG. Estudo do efeito da adição de soro de queijo na qualidade sensorial do doce de leite pastoso. Ciênc Tecnol Aliment. 2009;29(4):1-8. 